\title{
LOOK-AHEAD CONTROL FOR HEAVY TRUCKS TO MINIMIZE TRIP TIME AND FUEL CONSUMPTION
}

\author{
Erik Hellström* Maria Ivarsson ** Jan Åslund* \\ Lars Nielsen*
}

\author{
* Linköping University, Linköping Sweden \\ ** Scania CV AB, Södertälje Sweden
}

\begin{abstract}
The scenario studied is a drive mission for a heavy diesel truck. With aid of an on board road slope database in combination with a GPS unit, information about the road geometry ahead is extracted. This look-ahead information is used in an optimization of the velocity trajectory with respect to a criterion formulation that weighs trip time and fuel consumption. A dynamic programming algorithm is devised and used in a predictive control scheme by constantly feeding the conventional cruise controller with new set points. The algorithm is evaluated with a real truck on a highway, and the experimental results show that the fuel consumption can be significantly reduced. Copyright (c) 2007 IFAC
\end{abstract}

Keywords: predictive control, dynamic programming, fuel-optimal control

\section{INTRODUCTION}

As much as about $30 \%$ of the life cycle cost of a heavy truck comes from the cost of fuel. Further, the average mileage for a (European class 8) truck is $150,000 \mathrm{~km}$ per year and the average fuel consumption is $32.5 \mathrm{~L} / 100 \mathrm{~km}$ (Schittler, 2003). Lowering the fuel consumption with only a few percent will thus still translate into significant cost reductions. These facts makes a system which can reduce the fuel consumption appealing to owners and manufacturers of heavy trucks. The problem scenario in the present work is a drive mission for a truck where the route is considered to be known. It is however not assumed that the vehicle constantly operates on the same route. Instead, it is envisioned that there is road information on board and that the current heading is predicted or supplied by the driver. In the current work, information about the road slope is exploited aiming at a fuel consumption reduction.
One early work (Schwarzkopf and Leipnik, 1977) formulates an optimal control problem for a nonlinear vehicle model with the aim at minimizing fuel consumption and explicit solutions are obtained for constant road slopes. A dynamic programming approach is taken in Monastyrsky and Golownykh (1993) to obtain solutions for a number of driving scenarios on shorter road sections. Inspired of some of the results indicated in these and other works it was shown in Chang and Morlok (2005); Fröberg et al. (2006) with varying vehicle model complexity, that constant speed is optimal on a constant road slope within certain bounds on the slope. The result relies on that there is an affine relation between the fuel consumption and the produced work. Analytical studies of the situation when this relation is nonlinear is made in Fröberg and Nielsen (2007).

Predictive cruise control is investigated through computer simulations in e.g. Lattemann et al. (2004); Terwen et al. (2004) where in the latter 
paper, the solution of the optimal control problem is carried out by a combination of combinatorial search and a shooting algorithm. In Hellström et al. (2006) a predictive cruise controller is also developed where discrete dynamic programming is used to numerically solve the optimal control problem. The current paper is a continuation where an improved approach is realized and evaluated in actual experiments.

The prediction model in the present work is of hybrid nature and has time delays which turns the optimization into a challenging task. Further, constructing an optimizing controller that works on board in a real environment puts additional demands on the system in terms of robustness and complexity. Thus, the purpose of the present paper is to devise a control criterion on the basis of the objectives and to design a control algorithm by using a sufficiently complex system model, and further, to analyze controller behavior in trial runs and evaluate potential benefits.

The presentation is organized so that the prediction model used in the optimization algorithm is described first. Following that the control algorithm and the experimental setup are explained. Finally, results from the trial runs are presented and conclusions from the work are drawn.

\section{TRUCK MODEL}

A continuous-time dynamical model for the longitudinal dynamics of a truck is formulated first in this section (Kiencke and Nielsen, 2005; Sandberg, 2001). Thereafter the model is reformulated and adapted for the numerical optimization that is to be performed.

The engine torque $T_{e}$ is modeled as

$$
T_{e}\left(\omega_{e}, u_{f}\right)=a_{e} \omega_{e}+b_{e} u_{f}+c_{e}
$$

where $\omega_{e}$ is the engine speed and $u_{f}$ is the control signal which determines the fueling level.

The control $u_{f}$ is assumed to be bounded by

$$
0 \leq u_{f} \leq u_{f, \max }\left(\omega_{e}\right)
$$

where the upper limit $u_{f, \max }\left(\omega_{e}\right)$ is modeled by a second-order polynomial in engine speed $\omega_{e}$,

$$
u_{f, \max }\left(\omega_{e}\right)=a_{f} \omega_{e}^{2}+b_{f} \omega_{e}+c_{f} .
$$

When a gear is engaged, the engine transmits a torque $T_{c}$ to the clutch and

$$
J_{e} \dot{\omega}_{e}=T_{e}-T_{c}
$$

where $J_{e}$ is the engine inertia and $\omega_{e}$ is the engine speed. The clutch, propeller shafts and drive shafts are assumed stiff and their inertia are lumped into one together with the wheel inertia, denoted $J_{l}$. The resulting conversion ratio of the transmission and final drive is denoted $i$ and energy losses are modeled with an efficiency $\eta$. When a gear is engaged, this gives

$$
\begin{aligned}
\omega_{e} & =i \omega_{w} \\
T_{w} & =i \eta T_{c} \\
J_{l} \dot{\omega}_{w} & =T_{w}-T_{b}-r_{w} F_{w}
\end{aligned}
$$

where $T_{w}$ is the torque transmitted to the wheel, $T_{b}$ is the braking torque and $r_{w}$ is the wheel radius. $F_{w}$ is the resulting friction force.

When neutral gear is engaged, the engine transmits zero torque to the driveline. The driveline equations (3) and (4) then become

$$
J_{e} \dot{\omega}_{e}=T_{e}
$$

and

$$
\begin{aligned}
T_{c}=T_{w} & =0 \\
J_{l} \dot{\omega}_{w} & =-T_{b}-r_{w} F_{w} .
\end{aligned}
$$

The motion of the truck is governed by

$$
m \frac{d v}{d t}=F_{w}-F_{a}(v)-F_{r}(\alpha)-F_{N}(\alpha)
$$

where $\alpha$ is the road slope. The models of the longitudinal forces are explained in Table 1.

Table 1. Longitudinal forces.

\begin{tabular}{lll} 
Force & Explanation & Expression \\
\hline$F_{a}(v)$ & Air drag & $\frac{1}{2} c_{w} A_{a} \rho_{a} v^{2}$ \\
$F_{r}(\alpha)$ & Rolling resistance & $m g c_{r} \cos \alpha$ \\
$F_{N}(\alpha)$ & Gravitational force & $m g \sin \alpha$ \\
\hline
\end{tabular}

Gear shifts are assumed to be carried out by engine control, see e.g. (Pettersson and Nielsen, 2000). A shift is modeled by a constant delay time $\tau_{\text {shift }}$ where the neutral gear is engaged before the new gear is engaged. The number of the currently engaged gear will be denoted $g$. The ratio $i$ and efficiency $\eta$ then becomes functions of $g$. The control signal that selects gear will be denoted $u_{g}$. Neutral gear corresponds to gear zero, equivalent with a ratio and efficiency of zero.

The vehicle velocity $v$ is

$$
v=r_{w} \omega_{w}
$$

where $\omega_{w}$ is the wheel speed of revolution and $r_{w}$ is the effective wheel radius. Equation (3)-(8) can now be combined into 


$$
\begin{aligned}
& \frac{d v}{d t}(x, u, \alpha)= \\
& \frac{r_{w}}{J_{l}+m r_{w}^{2}+\eta(g) i(g)^{2} J_{e}}\left(i(g) \eta(g) T_{e}\left(v, u_{f}\right)\right. \\
& \left.-T_{b}\left(u_{b}\right)-r_{w}\left(F_{a}(v)+F_{r}(\alpha)+F_{l}(\alpha)\right)\right)
\end{aligned}
$$

where

$$
x=[v, g]^{T} \quad u=\left[u_{f}, u_{b}, u_{g}\right]^{T}
$$

denote the state and control vector respectively. The states are the velocity $v$ and currently engaged gear $g$ and the controls are fueling $u_{f}$, braking $u_{b}$ and gear $u_{g}$.

The mass flow of fuel is determined by the fueling level $u_{f}[\mathrm{~g} /$ stroke $]$ and the engine speed $\omega_{e}[\mathrm{rad} / \mathrm{s}]$. The flow in $[\mathrm{g} / \mathrm{s}]$ is then

$$
\frac{d m}{d t}\left(\omega_{e}, u\right)=\frac{n_{c y l}}{2 \pi n_{r}} \omega_{e} u_{f}
$$

where $n_{c y l}$ is the number of cylinders and $n_{r}$ is the number of crankshaft revolutions per cycle. Using (4) and (8) in (11) gives

$$
\frac{d m}{d t}(x, u)=\frac{n_{c y l}}{2 \pi n_{r}} \frac{i}{r_{w}} v u_{f}, g \neq 0
$$

whereas in the case of neutral gear, $g=0$, the fuel flow is assumed constant and equal to an idle fuel flow $\dot{m}_{i d l e}$.

\subsection{Reformulation}

The models (9) and (12) are transformed to be dependent on position rather than time. Denoting traveled distance with $s$ and the trip time with $t$, then for a function $h(t(s))$

$$
\frac{d h}{d s}=\frac{d h}{d t} \frac{d t}{d s}=\frac{1}{v} \frac{d h}{d t}
$$

is obtained using the chain rule where $v>0$ is assumed. By using (13), the models can be transformed as desired.

The approach in this work is numerical and therefore the model equations should be made discrete. The quantization step in position will be constant and equal to $h$. The control signals will be considered piece-wise constant during a discretization step. Denote

$$
\begin{aligned}
& x_{k}=x(k h), u_{k}=u(k h) \\
& \alpha_{k}=\frac{1}{h} \int_{k h}^{(k+1) h} \alpha(s) d s
\end{aligned}
$$

where the road slope $\alpha_{k}$ is set to the mean value over the discretization step. The trapezoidal rule is used to make the truck model (9) discrete. If a gear shift occurs during a step, a second-order Runge-Kutta method is used for a time step equal to the delay $\tau_{\text {shift }}$ to modify the initial values and the step length. The system dynamics is finally

$$
x_{k+1}=f\left(x_{k}, u_{k}, \alpha_{k}\right)
$$

where $f\left(x_{k}, u_{k}, \alpha_{k}\right)$ is given by (9).

The discretized problem will be incorporated into the algorithm and will thus affect the algorithm complexity. If speed rather than accuracy is wanted, the simplest methods would be tried. The simplest formula is Euler's method. It has been shown that for the current model description and objective this formula is not satisfactory due to truncation errors. For this reason the secondorder methods were chosen.

\section{LOOK-AHEAD CONTROL}

A model predictive controller (MPC) has been developed to control the vehicle. The approach is to first define a look ahead horizon and to solve the resulting optimization problem repeatedly online. The rationale is that it is not anticipated that the problem size will be manageable if the solution is to be calculated for the entire information horizon present, that is the entire route ahead which is known. The MPC principle is shown in Figure 1. At point $\mathrm{A}$, the state at point $\mathrm{B}$ is first predicted. During the travel time to $\mathrm{B}$, the optimal control is calculated with the look ahead horizon taken into account. When B is reached, the calculated control is output to the vehicle and the procedure restarts.

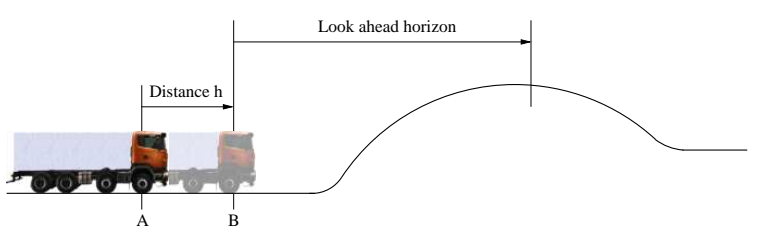

Figure 1. Controller principle.

This section will first deal with the identification of the control objectives. Based on these, a suitable criterion is devised and the tuning of its parameters is discussed. At the end, the dynamic programming (DP) algorithm will be explained.

\subsection{Objective}

The objectives are to minimize the energy and time required for a given drive mission. The vehicle is desired to be kept inside an interval

$$
v_{\min } \leq v \leq v_{\max }
$$


where $v$ denotes the vehicle velocity. These bounds are set with respect to the desired behavior of the controller. For example, the lower bound will be the lowest velocity the controller would deliberately actuate. The upper bound can be set by e.g. safety reasons or legal considerations.

The brake system is assumed to be powerful enough to keep the upper bound in (16). On the other hand, the lower bound is not expected to be physically reachable on all road profiles. It is assumed though, that it is possible to keep a velocity, denoted $v_{l i m}$, which is greater than zero at all times. If Equation (16) was to be used, it would not be certain to find any feasible solution. Therefore the constraints on the vehicle speed $v$ are expressed as follows.

$$
0<\min \left\{v_{\min }, v_{\text {lim }}(s)\right\} \leq v \leq v_{\max }
$$

\subsection{Criterion}

The fundamental trade off when studying minimization of energy required for a drive mission is between the fuel use and the trip time. The fuel use on a trip from $s=s_{0}$ to $s=s_{f}$ is

$$
M=\int_{s_{0}}^{s_{f}} \frac{d m}{d s}(v, u) d s
$$

where $\frac{d m}{d s}(v, u)$ is the mass flow per unit length as function of the state $v$ and control $u$. The trip time $T$ is simply

$$
T=\int_{s_{0}}^{s_{f}} \frac{d t}{d s} d s=\int_{s_{0}}^{s_{f}} \frac{d s}{v}
$$

To weigh fuel and time use, the cost function proposed is

$$
I=M+\beta T
$$

using (18) and (19) and where $\beta$ is a scalar factor which can be tuned to receive the desired trade off.

The criterion (20) is then made suitable for discrete dynamic programming by dividing the look ahead horizon into $N$ steps of length $h[\mathrm{~m}]$ and transforming the cost function. Denote

$$
\begin{aligned}
m_{k} & =\int_{k h}^{(k+1) h} \frac{d m}{d s}(x, u) d s, \quad t_{k}=\int_{k h}^{(k+1) h} \frac{d s}{v}, \\
a_{k} & =\left|v_{k}-v_{k+1}\right|
\end{aligned}
$$

and the cost function can be expressed as

$$
J=\sum_{k=0}^{N-1} \zeta_{k}\left(x_{k}, x_{k+1}, u_{k}, \alpha_{k}\right)
$$

where

$$
\begin{gathered}
\zeta_{k}=[1, \beta, \gamma]\left[\begin{array}{c}
m_{k} \\
t_{k} \\
a_{k}
\end{array}\right] \\
k=0,1, \ldots, N-1
\end{gathered}
$$

and $\beta, \gamma$ are scalar penalty parameters for controlling the properties of solutions. The term $a_{k}$ was added to enable smoothing of the solution.

\subsection{Penalty parameters}

One way to determine the parameter $\beta$, i.e. the trade off between fuel and time, is to study a stationary solution to the criterion in Equation (20).

Assume that a gear is engaged and there exists at least one control $\hat{u}$, for which (2) holds and that gives a stationary velocity $\hat{v}$. From the equations (1), (9) and Table 1 it is concluded that $\hat{u}$ can be written as

$$
\hat{u}=c_{1} \hat{v}^{2}+c_{2} \hat{v}+f(\alpha)
$$

where, for a given gear, $c_{1}, c_{2}$ are constants and $f(\alpha)$ is a function of the road slope $\alpha$ corresponding to the rolling resistance, gravity and engine friction. With (12) and (13), the fuel flow is written as

$$
\frac{d m}{d s}(x, u)=c_{4} u_{f}
$$

where $c_{4}$ is the proportionality constant. The cost function (20) is thus

$$
\hat{I}(\hat{v})=\int_{s_{0}}^{s_{f}}\left(c_{4}\left(c_{1} \hat{v}^{2}+c_{2} \hat{v}+f(\alpha)\right)+\frac{\beta}{\hat{v}}\right) d s
$$

where the integrand is constant with respect to $s$ if constant slope is assumed. A stationary point to $\hat{I}$ is found by setting the derivative equal to zero,

$$
\frac{d \hat{I}}{d \hat{v}}=\int_{s_{0}}^{s_{f}}\left(c_{4}\left(2 c_{1} \hat{v}+c_{2}\right)-\frac{\beta}{\hat{v}^{2}}\right) d s=0 .
$$

Solving the equation for $\beta$ gives

$$
\beta=c_{4} \hat{v}^{2}\left(2 c_{1} \hat{v}+c_{2}\right)
$$

which can be interpreted as the required value of $\beta$ to achieve the stationary velocity $\hat{v}$ for a fixed slope. The value of $\beta$ neither depends on the vehicle mass nor the slope and will thus give the stationary velocity $\hat{v}$ for any fixed mass and slope as long as there exists a control $\hat{u}$ satisfying (2). 


\subsection{Preprocessing}

The ambition with the present work is a realtime algorithm and hence the complexity plays an important role. The subset of the state space over which the optimization is applied, the search space, is one determining factor for the complexity. If the search space is reduced without loosing any solutions, obvious gains are made. A preprocessing algorithm is therefore developed with this aim.

Since DP is used in an MPC setting, the current velocity can be measured and used for limiting the set of possible initial states. In order to handle terminal effects, the final velocities are also constrained. By using the model and traversing the horizon forward and backward before the optimization is started, the search space is downsized.

\subsection{DP algorithm}

To summarize, the optimal control problem at hand is the minimization of the objective,

$$
\min _{u, g} \sum_{k=0}^{N-1} \zeta_{k}\left(x_{k}, x_{k+1}, u_{k}, \alpha_{k}\right)
$$

where $\zeta_{k}$ is given in (23). The system dynamics is given by

$$
x_{k+1}=f\left(x_{k}, u_{k}, \alpha_{k}\right) \quad k=0,1, \ldots, N-1
$$

according to (15). The constraints are

$$
0<\min \left\{v_{\min }, v_{l i m}(k h)\right\} \leq v_{k} \leq v_{\max } \forall k
$$

according to (17). Due to the MPC setting, the initial state $x_{0}$ is given.

The preprocessing algorithm gives, for each stage, an interval of velocities which are to be considered. For every stage the interval $\left[v_{l o}, v_{u p}\right]$ is discretized in constant steps of $\delta$. This makes up a set $V_{k}$,

$$
V_{k}=\left\{v_{l o}, v_{l o}+\delta, v_{l o}+2 \delta, \ldots, v_{u p}\right\} .
$$

With a given velocity, only a subset of the gears in the gearbox is feasible. If the operating region of the engine is defined with bounds on the engine speed $\left[\omega_{e, \min }, \omega_{e, \max }\right]$, it is easy to select the set of feasible gears. Only gears with a ratio that gives an engine speed in the allowed range are then considered. In a state with velocity $v$, the set of usable gears $G_{v}$ is thus defined as

$$
G_{v}=\left\{g \mid \omega_{e, \min } \leq \omega_{e}(v, g) \leq \omega_{e, \max }\right\}
$$

where $\omega_{e}(v, g)$ is the engine speed at vehicle velocity $v$ and gear number $g$.
Table 2. Truck specifications.

\begin{tabular}{rll} 
Component & Type & Characteristics \\
\hline Engine & DC9 & $\begin{array}{l}\text { cylinders: } 5 \\
\text { displacement: } 9 \mathrm{dm}^{3} \\
\end{array}$ \\
& & $\begin{array}{l}\text { max.torque: } 1,550 \mathrm{Nm} \\
\text { max.power: } 310 \mathrm{Hp}\end{array}$ \\
& & 12 gears \\
Gearbox & GRS890R & total weight: $39,410 \mathrm{~kg}$
\end{tabular}

The possible states $x=[v, g]^{T}$ in stage $k$ is a set $S_{k}$ and will be generated from the velocity range $V_{k}$ given in (29) and the set of gears $G_{v}$ given in (30). This yields

$$
S_{k}=\left\{\{v, g\} \mid v \in V_{k}, g \in G_{v}\right\} .
$$

At a stage $k$, feasible control actions $u_{k}^{i, j}$ that transform the system from a state $x^{i} \in S_{k}$ to another state $x^{j} \in S_{k+1}$ are sought. The control is found by an inverse simulation of the system equations. If there are no fueling level $u_{f}$ and gear $u_{g}$ that transforms the system from state $x^{i}$ to $x^{j}$ at stage $k$, there are two possible resolutions. If there exist a feasible braking control $u_{b}$ the cost of the transition is set accordingly. If there is no feasible braking control the cost is set to infinity, that with a numerical approach means a very large number. The algorithm is outlined below.

(1) Let $J_{N}(i)=0$.

(2) Let $k=N-1$.

(3) Let

$$
J_{k}\left(x^{i}\right)=\min _{x^{j} \in S_{k+1}}\left\{\zeta_{k}^{i, j}+J_{k+1}\left(x^{j}\right)\right\}, x^{i} \in S_{k} .
$$

(4) Repeat (3) for $k=N-2, N-3, \ldots, 0$.

(5) The optimal cost is $J_{0}$ and the sought control is the optimal control set from the initial state.

\section{TRIAL RUN}

The experiments are performed on the highway E4 between the cities of Södertälje and Norrköping in Sweden, see Figure 3. The truck used is a SCANIA tractor and trailer that has the specifications according to Table 2 .

Following in this section, the experimental setup and road slope estimation will be explained. The last part of the section will present some results from the trial runs that have been undertaken.

\subsection{Setup}

The information flow in the experimental setup is shown schematically in Figure 2. In this first trial run, it is decided not to control the gear selection. Gear shifting is fully controlled by the SCANIA system for automatic gear shifting of 


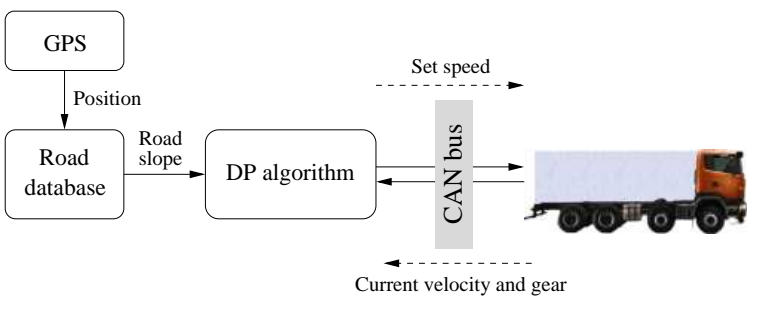

Figure 2. Information flow.

Table 3. User parameters

\begin{tabular}{clc} 
Parameter & Function & Value \\
\hline$h$ & Step length & $50 \mathrm{~m}$ \\
$N$ & Number of steps & 30 \\
$h \cdot N$ & Horizon & $1500 \mathrm{~m}$ \\
$\delta$ & Velocity discretization & $0.2 \mathrm{~km} / \mathrm{h}$ \\
$v_{\min }$ & Min. allowed vel. & $79 \mathrm{~km} / \mathrm{h}$ \\
$v_{\max }$ & Max. allowed vel. & $89 \mathrm{~km} / \mathrm{h}$
\end{tabular}

Table 4. Penalty factors

\begin{tabular}{clc} 
Factor & Penalizes & Value \\
\hline & Fuel use & 1.0 \\
$\beta$ & Time use & 6.2 \\
$\gamma$ & Velocity changes & 0.1
\end{tabular}

manual gearboxes. This is handled by making a simple model of the shift control system and take it into account when calculating the transition cost $\zeta$ in (22). In case the simplified model does not predict the gear shift from the cost is set to infinity. As depicted in Figure 2 the algorithm controls the vehicle by adjusting the set speed to the conventional cruise controller. The fueling level is therefore only controlled indirectly. All communication is done over the CAN bus.

The algorithm parameters used are stated in Table 3 and the penalty factors are shown in Table 4. The factors are adjusted in order to receive a stationary solution in the middle of the desired velocity interval (16). All software run on a portable computer with an Intel Centrino Duo processor at $1.20 \mathrm{GHz}$ and $1 \mathrm{~Gb}$ RAM. With the stated parameters, a solution on a road stretch of level road is calculated in less than about $0.3 \mathrm{~s}$ which is equivalent to about $7.4 \mathrm{~m}$ traveling in $89 \mathrm{~km} / \mathrm{h}$.

The truck has a legislative speed limiter at 89 $\mathrm{km} / \mathrm{h}$. Propulsion above this limit is not possible. When the truck accelerates due to gravity above $89 \mathrm{~km} / \mathrm{h}$, the brake control system is activated at a set maximum speed. In the trial run this limit is set to be $91 \mathrm{~km} / \mathrm{h}$.

Database The slope in front of the vehicle for the length of the look-ahead horizon is needed to be known in advance. For this reason, the road slope along the trial route is estimated off line prior to any experiments. This is done by aid of a non-stationary forward-backward Kalman filter (Sahlholm et al., 2007). The estimated slope and calculated altitude are shown in Figure 3. The measurements were obtained at $20 \mathrm{~Hz}$ from a GPS unit. The filter inputs are vertical and horizontal velocity of the vehicle, altitude and the number of reachable GPS satellites.

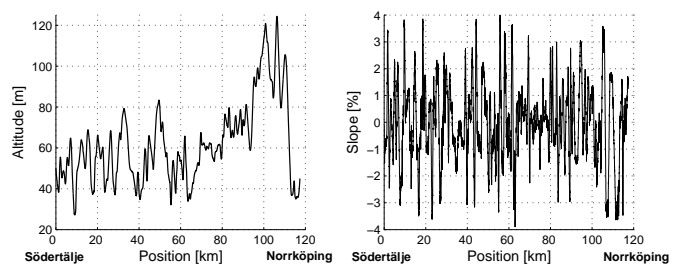

Figure 3. Estimated road topography.

\subsection{Performance}

In total, five comparative trial runs were made. All runs were done in light to moderate traffic, and each consisted of one drive with look-ahead control and one with standard cruise control in a direction on the trial route. The algorithm parameters, see Table 3 and 4 , were the same for all runs. The trip time will then become about the same for all drives with the look-ahead control. The set point for the cruise controller was varied in order to receive a trip time close to the one obtained with look ahead.

\subsection{Overall results}

The relative change in fuel consumption and trip time ( $\Delta$ fuel, $\Delta$ time) are shown in Figure 4 and Figure 5 for each direction on the trial road. A negative value means that the lookahead controller (MPC) has reduced the corresponding value. The set point for the cruise controller (CC) increases along the horizontal axis.

The average results in both directions that are

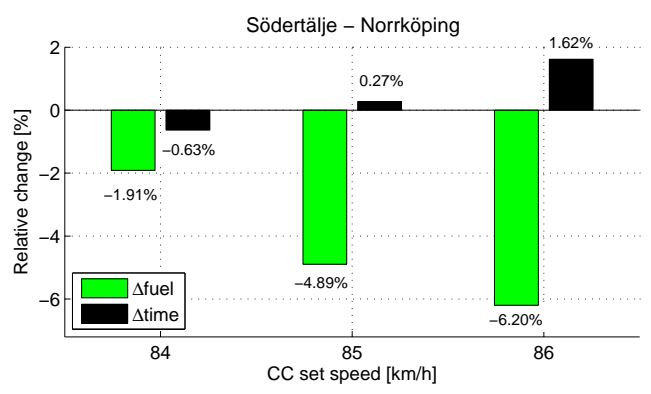

Figure 4. Trial run results on the road from Södertälje to Norrköping with varying cruise controller (CC) set speed.

made with the same set speed are also calculated. These show that the fuel consumption could be decreased with $3.53 \%$, from $36.33 \mathrm{~L} / 100 \mathrm{~km}$ to $35.03 \mathrm{~L} / 100 \mathrm{~km}$, with a negligible reduction of the trip time (0.03\%) in comparison with the CC. Also 


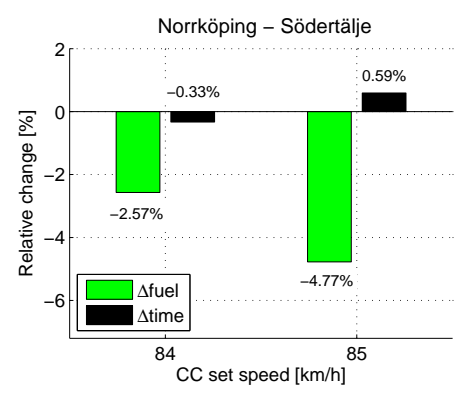

Figure 5. Trial run results on the road from Norrköping to Södertälje with varying cruise controller (CC) set speed.

interesting to note is that the mean number of gear shifts on this route decreases from 20 to 12 $(-42 \%)$ with the MPC.

\subsection{Control characteristics}

Detailed controller behavior will be presented in each direction for one selected section of the trial route. Each figure is divided into four subfigures, see e.g. Figure 6, all having position as the horizontal axis. The altitude is shown at the top where the coordinates for the start and final position are given at the horizontal axis. The next subfigure shows the velocity trajectories for the look-ahead controller (MPC) and the standard cruise controller (CC). In the third part normalized fueling (acc) and retarder (brake) levels are shown with thick and thin lines respectively. At the bottom, both the engaged gear number and the fuel use are shown. Data related to the MPC is displayed in solid lines and data associated to the $\mathrm{CC}$ is displayed with dashed lines consistently in these figures. Above the figures, the time and fuel spent on the section are shown.

Figure 6 shows a piece of about $3.5 \mathrm{~km}$ from about halfway between Södertälje and Norrköping. At $500 \mathrm{~m}$ it is seen that the MPC accelerates prior to the uphill which begins at $750 \mathrm{~m}$. This leads to a higher velocity climbing the hill. The CC will of course keep its set point running into the hill and has therefore a lower velocity and is also spending slightly more time on the lower gear. At the top of the hill at $1750 \mathrm{~m}$, the MPC slows down in contrast to the CC. The truck is thus let to accelerate by the slope. The $\mathrm{CC}$ will however use a non-zero fueling as long as the truck goes slower than the set point. This reduces the need for braking later in the downslope and thereby the inherent waste of energy is lessened. Looking at the fuel integral at the bottom, it is seen that the MPC consumes more fuel the first $1.5 \mathrm{~km}$ owing to the acceleration. However, in total less fuel is spent by the MPC due to the reduced fueling at the top of the hill.
Traveling in the other direction, see Figure 7, gives similar features. A gain of speed at $250 \mathrm{~m}$ and then a reduced fueling at the top of the hill at $2250 \mathrm{~m}$. In both directions, time as well as fuel are saved.
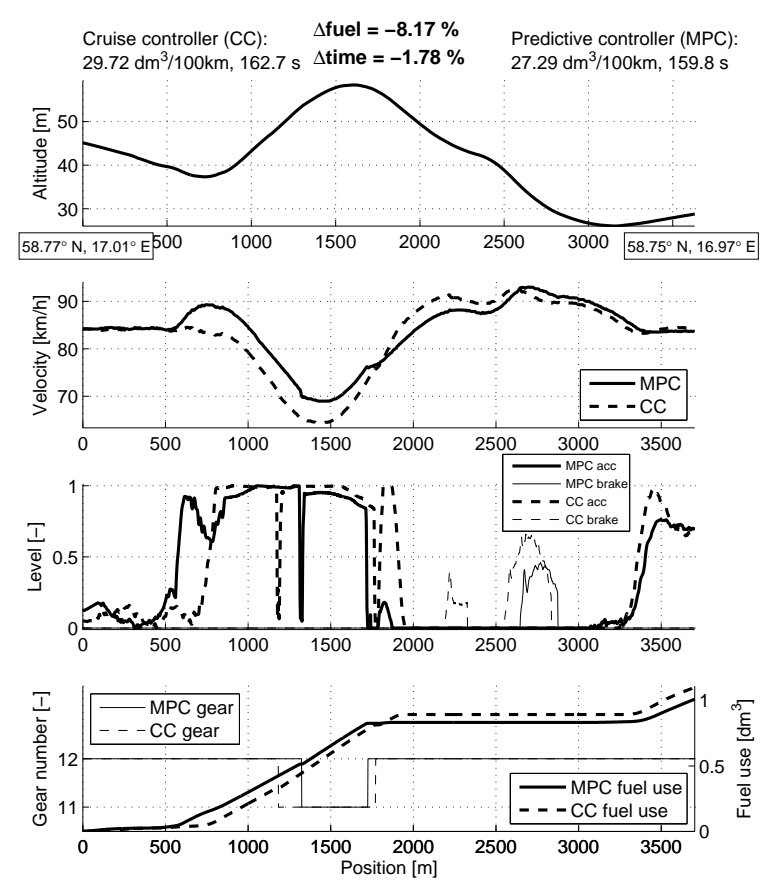

Figure 6. A section of the road from about halfway between Södertälje and Norrköping. The MPC accelerates at $500 \mathrm{~m}$ prior to the uphill and slows down at $1750 \mathrm{~m}$ when the top is reached.
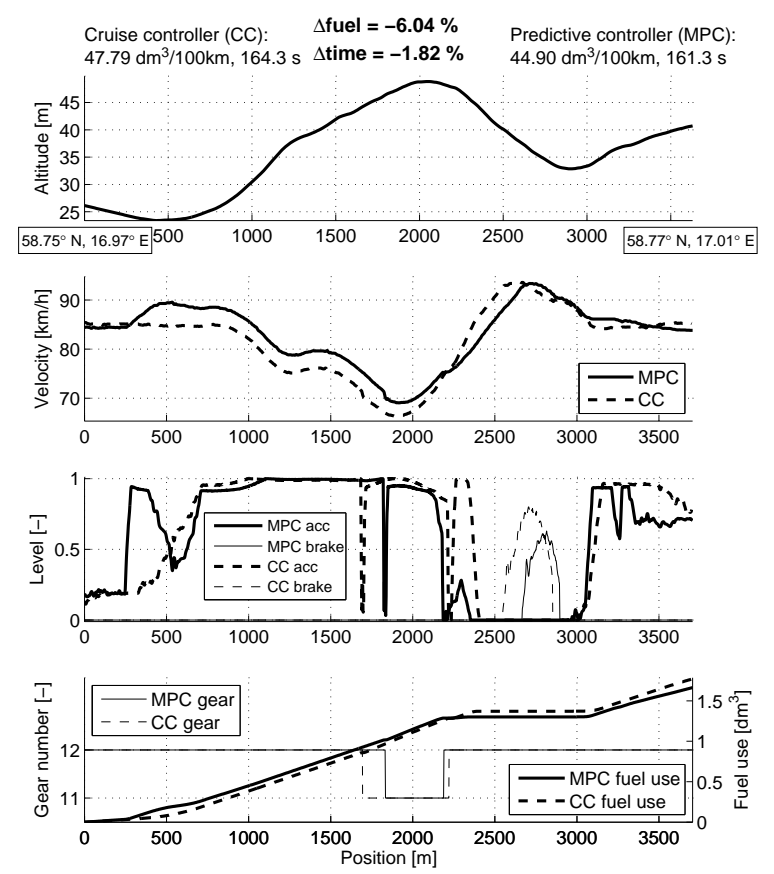

Figure 7. A section of the road from about halfway between Södertälje and Norrköping. The MPC gains speed at $250 \mathrm{~m}$ prior to the uphill and slows down at $2250 \mathrm{~m}$ prior to the downhill. 
Note that the sections in Figure 6 and 7 are not exceptionally steep. The uphill and downhill slope is at most about $4 \%$ for short intervals. However, they become significant for the truck due to the large vehicle mass.

\section{CONCLUSIONS}

The control algorithm was proven to perform well on board in a real environment. Using the standard cruise controller as an inner loop and constantly feeding it with new set points is advantageous considering robustness against model errors and disturbances.

Modeling the gearbox as a set of discrete gears and the shift process as a time delay renders a challenging optimization problem. With careful discretization of the model equations, dynamic programming is applied. The search space is reduced by a preprocessing algorithm. Owing to this design, a solution is calculated in tenths of a second on a modern PC and that allowed evaluation in a real environment on board a truck.

The trial runs show that significant reductions of the fuel consumption can be achieved. On the entire route of about $120 \mathrm{~km}$ a $3.5 \%$ fuel use reduction without an increase in trip time was obtained. The mean number of gear shifts was reduced with $42 \%$ due to shifts avoided by gaining speed prior to uphills.

The look-ahead control mainly differs from conventional cruise control near significant downhills and uphills where the look-ahead control in general slows down or gains speed prior to the hill. Slowing down prior to downhills is intuitively saving fuel. There is however no challenge in saving fuel by traveling slower, so if the vehicle is let to slow down at some point, the lost time must thus be gained at another point. Accelerating prior to uphills is one way which, at least for shorter hills, gives a higher velocity throughout the hill and will reduce the need for lower gears.

A final comment is that the controller behavior has been perceived as comfortable and natural by drivers and passengers that have participated in tests and demonstrations.

\section{REFERENCES}

Chang, D. J. and E. K. Morlok (2005). Vehicle speed profiles to minimize work and fuel consumption. Journal of transportation engineering 131(3), 173-181.

Fröberg, A. and L. Nielsen (2007). Optimal fuel and gear ratio control for heavy trucks with piece wise affine engine characteristics. 5th IFAC Symposium on Advances in Automotive Control. Monterey, CA, USA.

Fröberg, A., E. Hellström and L. Nielsen (2006). Explicit fuel optimal speed profiles for heavy trucks on a set of topographic road profiles. number 2006-01-1071 In: SAE World Congress. Detroit, MI, USA.

Hellström, E., A. Fröberg and L. Nielsen (2006). A real-time fuel-optimal cruise controller for heavy trucks using road topography information. number 2006-01-0008 In: SAE World Congress. Detroit, MI, USA.

Kiencke, U. and L. Nielsen (2005). Automotive Control Systems, For Engine, Driveline, and Vehicle. 2nd ed.. Springer Verlag.

Lattemann, F., K. Neiss, S. Terwen and T. Connolly (2004). The predictive cruise control - a system to reduce fuel consumption of heavy duty trucks. SAE Technical paper series 200401-2616.

Monastyrsky, V.V and I.M. Golownykh (1993). Rapid computations of optimal control for vehicles. Transportation Research 27B(3), 219227.

Pettersson, M. and L. Nielsen (2000). Gear shifting by engine control. IEEE Transactions on Control Systems Technology 8(3), 495-507.

Sahlholm, P., H. Jansson, E. Kozica and K.H. Johansson (2007). A sensor and data fusion algorithm for road grade estimation. 5th IFAC Symposium on Advances in Automotive Control. Monterey, CA, USA.

Sandberg, Tony (2001). Simulation tool for predicting fuel consumption for heavy trucks. IFAC Workshop: Advances in Automotive Control. Karlsruhe, Germany.

Schittler, M. (2003). Diesel engine emissions reduction conference. U.S. Department of Energy. Rhode Island, USA.

Schwarzkopf, A.B. and R.B. Leipnik (1977). Control of highway vehicles for minimum fuel consumption over varying terrain. Transportation Research 11(4), 279-286.

Terwen, S., M. Back and V. Krebs (2004). Predictive powertrain control for heavy duty trucks. 4th IFAC Symposium on Advances in Automotive Control. Salerno, Italy. 